\title{
Comparison of Pre-Processing Methods for Segmentation and Approximation of Optic Disc Boundary From Processed Digital Retinal Images
}

\author{
YOGITA M. VAIDYA ${ }^{1}$, SUNIL EKNATH DOIPHODE ${ }^{2}$
}

\author{
${ }^{1}$ Assistant professor, Department of Electronics and Telecommunication Engineering, College of Engineering Pune \\ (COEP), Pune-411005, Maharashtra, India \\ ${ }^{2}$ Research Student, Department of Electronics and Telecommunication Engineering, College of Engineering Pune \\ (COEP), Pune-411005, Maharashtra, India
}

Yogita M. Vaidya: m_yogita@yahoo.co.in

\begin{abstract}
Algorithms are presented for rapid, automatic and accurate segmentation and Approximation of Optic Disc boundary from digital retinal images. This paper presents method that improves upon prior work in different ways; 1.Accuracy 2.Contrast. The pre-processing methods are presented here to enhance the image properties prior to the segmentation of OD from digital fundus images of retina using template based methodology. This paper compares performance of contrast enhancement techniques such as Intensity thresholding, adaptive histogram equalization and histogram equalization. Further, evaluates performance of each of these techniques with respect to the original template based OD segmentation using circular Hough Transform. Intensity thresholding method provides better performance with Maximum Variance method and Low Pass Filter method of ODP location. Histogram Equalization performs better with only Low Pass Filter method of ODP location. Adaptive Histogram Equalization performs better with all three methods of OD pixel location so ultimately it performs better with voting type algorithm that is used to locate the centroid of the pixel location detected by Maximum Difference; Maximum Variance and Low Pass Filter method. Thus the optic disc segmentation performance is improved over the original method. Experimental results on a known MESSIDOR database and local NIOP database, achieving to more than $93 \%$ accuracy for optic disc boundary approximation. it proved that adaptive histogram performs better as a contrast enhancement technique.
\end{abstract}

Keywords - Centroid, Contrast Enhancement, Diabetic Retinopathy (DR), Fast Fourier Transform (FFT), Hough Transform, Location Methods, Optic Disc (OD), Segmentation

\section{Introduction}

Glaucoma is not just an eye disease, but a group of eye conditions which can cause optic nerve damage and further it may cause loss of vision (blindness). It is predicted that approximately 80 million people of the world population will be affected by this disease in next 5-6 years. Experts have already studied and assessed benefits provided by a system which automatically detects symptoms of this disease. Thus Optic Disc plays an significant role in development of automated systems for diagnosis of glaucoma disease. OD segmentation is a important component in many feature extraction algorithms. These algorithms are implemented in order to identify other fundus features such as vascular tree, fovea. Many ophthalmic pathologies also used OD segmentation as key element for automated diagnosis [3],[4]. OD segmentation can be used for detecting early signs of ophthalmic disease such as diabetic retinopathy.

The OD is slightly elliptical in shape and it is easy to distinguish $\mathrm{OD}$ in digital fundus images. It may vary significantly in size depending on various factors such as age, gender of person. Sinthanayothin et. al [1] pointed out that OD covers about $15 \%$ area of overall area of the image, while other researchers stated that size of OD varies from person to person occupies about $10 \%$ to $20 \%$ area of an entire image.

OD segmentation involves lots of challenges mainly due to various cases such as occlusion of blood vessel, variations in image near the disk boundaries due to changes in pathological and imaging conditions, ill defined boundaries of OD. In general, similar region occurrence near boundary of OD, asymmetrical shape and irregular boundary are the important factors to be considered by method used for segmentation of OD. To improve the segmentation results, we have proposed contrast enhancement techniques as pre-processing component.

This paper presents Contrast Enhancement Techniques followed by OD segmentation using template based methodology. Proposed work flow will take place as follows, Firstly, image enhancement of original image is carried out using contrast enhancement techniques to get three enhanced images, then from these enhanced images, sub-image is extracted. This sub-image is selected in such a way that it includes whole OD with wide enough surrounding region. Proposed OD location methods are used for ODP location. Then morphological and edge detection techniques are used for extraction of OD boundary from green channel of extracted sub image. Circular Hough Transform is used for OD boundary approximation. The results of this study are used to verify 
suitability of contrast enhancement techniques for OD boundary segmentation in view of digital fundus images of Indian population.

\section{Literature Survey}

The initial research of OD processing in digital images of retina is classified into OD location methodology and OD segmentation methodology. The previous research concentrates on determining center pixel of OD. whereas the latter work focus on OD boundary estimation. For exact extraction the OD boundary, we can make general distinction among deformable model based method and template based method. Synthanayothin et al. presented an algorithm considering location methods [1], [5] where the local contrast of images were enhanced using the HIS color space intensity channel. Using the intensity variations produced due to blood vessels in OD region, location of center of OD was identified. Hoover and Goldbaum [6], [7] used vasculature origin technique to locate an OD center. They determined fuzzy convergence phenomenon of vessels. Foracchia et al [8] proposed method that involves vessels convergence phenomenon. Youssif et al [9] pointed out vessel direction matched filter based method for OD location.

With regard to segmentation methods based on deformable model, Osareh et al [10] proposed a template based method for location of OD center and for OD boundary extraction used initialization of snake on a OD region which was enhanced using morphological methods. Lowell used template matching for locating OD and for OD segmentation he used deformable contour model. Walter and Klein used watershed transformation for OD contour.

\section{Methodology}

The objective of proposed methodology is to validate the performance of the preprocessing technology with respect to the OD segmentation methodology that approximate OD boundary from retinal fundus image.

\section{A. Pre-processing (Contrast Enhancement)}

Image enhancement of original image is carried out using Contrast enhancement techniques. It consists of three different methods. Each method has its own significance. These methods are illustrated in Fig. 1 and description of these methods is presented as follows.

a. Contrast Enhancement using Intensity thresholding technique

In this, contrast enhancement of the image takes place by assigning new values to the input intensity image values. Input data with low and high intensity values are saturated by $1 \%$.

b. Histogram Equalization technique

In this, contrast enhancement of the image takes place by values transformation in an intensity image, or the color map values of an indexed image, such that histogram of output image approximately matching a specified histogram. The formula for histogram equalization is as stated below

$$
h(v)=\operatorname{round}\left(\frac{c d f(v)-c d f \min }{(M X N)-c d f \min } x(L-1)\right)
$$

Where, $M \times N$ is image resolution and $\mathrm{L}$ is gray level considered to be 256 in the present case.

c. Adaptive Histogram Equalization technique Histogram equalization enhances the contrast of the image globally. Whereas, modified histogram equalization called as Adaptive Histogram Equalization (AHE). it is used for local contrast enhancement of the image regions. The main difference between histogram equalization and adaptive histogram equalization is that adaptive method evaluates multiple histograms each corresponding to the distinct image sections and these are used in the redistribution of intensity values. That's why; AHE is best method for local contrast enhancement of an image and to improve visual appearance of image. AHE also has some additional features such as noise over amplifying tendency in relatively homogeneous image regions. The grid size is calculated based on the maximum dimension of the image. The following equation (2) is applied on the 32 pixels square grid size.

$$
h(v)=\operatorname{round}\left(\frac{c d f(v)-c d f \min }{(M X N)-c d f \min } x(L-1)\right)
$$

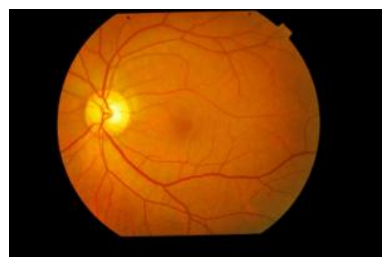

(a)

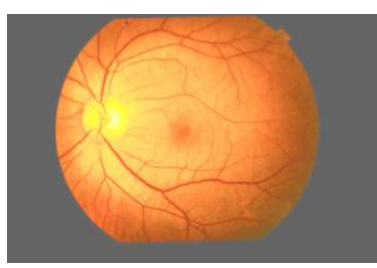

(c)

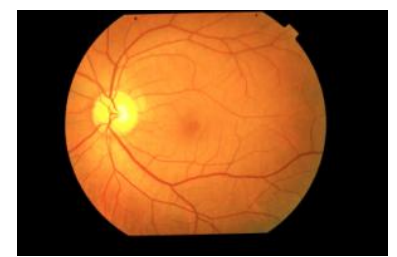

(b)

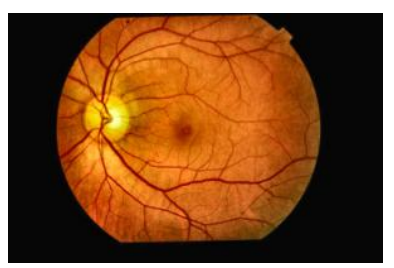

(d)
Fig. 1. Result of contrast enhancement techniques. (a)Input image (b)enhanced image using intensity thresholding contrast enhancement technique (c)enhanced image using histogram equalization contrast enhancement technique (d)enhanced image using adaptive histogram equalization contrast enhancement technique 


\section{B. Optic Disc Location}

Optic Disc Pixel (ODP) is obtained using location methodology. ODP is a pixel that belongs to an OD. Location methodology consists of 3 independent methods. As a result, each method gives OD candidate pixel. these three candidate OD pixels and their locations are considered for final ODP selection. For final ODP selection, a voting procedure is applied, it consists of the following cases

- If all three OD Pixel candidates are located close to the centroid then final OD pixel is the centroid.

- If only two OD Pixel candidates are located close to the centroid then final OD pixel is two referred pixels candidates average.

- Else, the final OD pixel is most reliable methods OD pixel candidate.

The three ODP location methods provide the best contrast as these methods work on the RGB color space. In this case, it used green channel of it. Let, I be the gray scale image. These methods are shown in Fig. 2 and these methods are described as follows;

- Maximum Difference method: The OD is characterized by a bright region in digital images of retina. the vascular tree emerges in the OD. It is formed due to the dark blood vessels convergence. That's why an occurrence of maximum gray level takes place within the OD pixel. Non significant peaks in the image are removed by applying. median filter of $21 \times 21$ to I If $\mathrm{I}_{\mathrm{M}}$ denotes this filtered image, this method uses the following equation(3) to decide OD pixel.

$$
O D P=\max \left(\left(I_{M}\right) W^{M A X}(i, j)-\left(\left(I_{M}\right) W^{M I N}(i, j)\right)\right)
$$

Where, $\left(I_{M}\right) W^{M A X}(i, j)$ and $\left(I_{M}\right) W^{M A X}(i, j)$ are the maximum and minimum pixel values within a $21 \times 21$ window size respectively.

- Maximum Variance Method: it uses the properties of maximum difference method. In this, the statistical variance is calculated for each pixel using $21 \times 21$ centered windows. An automated blue channel thresholding is used to obtain "bright" pixels set using canny edge detection. The selection of window size done for the variance computation and for the neighbor hood criteria establishment.

$$
\sigma(x, y)=\frac{1}{M N} \sum_{x=0}^{M} \quad \sum_{y=0}^{N}[f(x, y)-m]^{2}
$$

- Low pass filter method: The pixel with maximum gray level in an filtered image (low pass filter) is considered as OD pixel of this method. Although OD appears to be brightest area in a retinal images. the highest gray level pixel could not be appeared within OD. Most of the time, this highest gray level pixel may be inside other small bright regions. So the image I undergoes frequency domain transformation and Gaussian low pass filter is used to remove the distracters. Gaussian lpf defined as follows:

$$
H(u, v)=\exp ^{\left(\frac{-D(u, v)}{2 D_{0}{ }^{2}}\right)}
$$

Where, D $(\mathrm{u}, \mathrm{v})$ represents Euclidean distance between frequency plane origin and the point $(\mathrm{u}, \mathrm{v})$ and $\mathrm{D}_{0}$ is the cut-off frequency having value of 25 $\mathrm{Hz}$. The result of lowpass filter method of ODP location is the returned pixel with highest gray level to spatial domain.

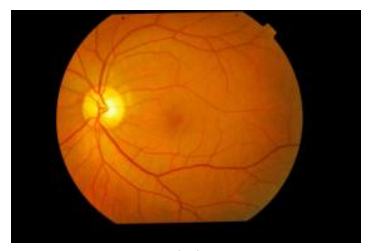

(a)

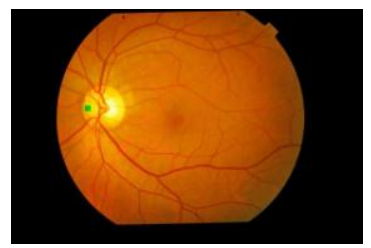

(c)

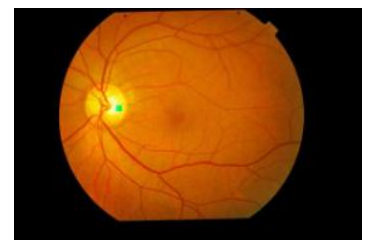

(b)

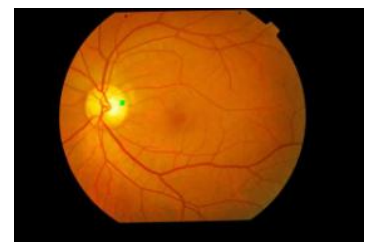

(d)

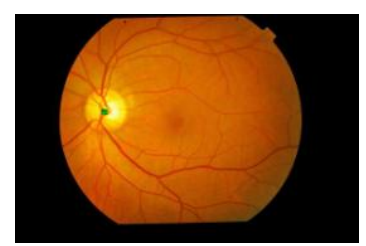

(e)

Fig. 2. Result of pixel location methodologies. (a)Input image (b)Image highlighting ODP located by maximum difference method (c)Image highlighting ODP located by maximum variance method (d)Image highlighting ODP located by low pass filter method

(e)Final OD selection by voting tvpe algorithm

The final ODP determination process is shown in Fig. 2. It highlights pixel location obtained by maximum difference, maximum variance and Low Pass filter methods without preprocessing as prescribed in the original reference work. Out of three OD candidate pixels, only two OD pixels are located close in vicinity of centroid, so the location of the OD pixel is two referred pixel candidates average.

These results can be improved by pre-processing the original image. These pre-processing methods enhance various image properties such as contrast, hue, brightness etc. This research work mainly focuses on implementation of contrast enhancement methods on digital fundus image before undergoing segmentation and boundary approximation algorithms. Figure 3 illustrates comparison of ODP location of three pre-processed images with the ODP location of original image (without preprocessing). 


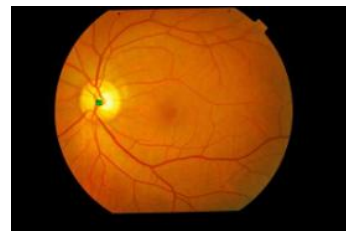

(a)

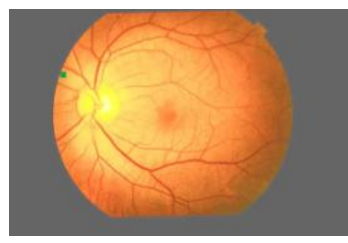

(c)

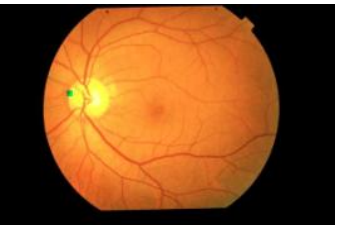

(b)

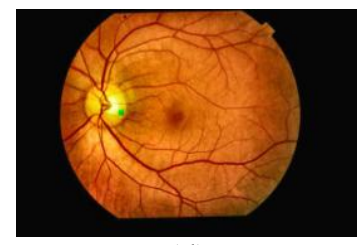

(d)
Fig. 3. Comparison of ODP location selection by voting type algorithm (a)ODP selection without preprocessing (b)ODP selection for pre-processed image (intensity thresholding) (c)ODP selection for pre-processed image (histogram equalization) (d)ODP selection for pre-processed image (adaptive histogram equalization)

\section{Optic Disc Boundary Segmentation}

The proposed method is applied to color sub-image extracted from original retinal image. This results in robust and efficient segmentation of an OD. It also introduces decrement in number of artifacts and reduction in the search space. We can apply proposed method on color subimage extracted from the original retinal image which reduces the search area and no. of distracters within the image. Firstly, a $150 \times 150$ RGB sub-image is extracted from original image. Extracted image having center as OD pixel provided by voting type algorithm. Then blood vessels are removed using morphological methods. After this, edge detection techniques are applied to obtain a binary mask. Finally, OD boundary approximated using circular Hough transform.

\section{$D$. Elimination of Blood vessel}

Consider sub-image containing the OD. Blood vessels within the OD are strong distracters, so it is necessary to remove from the image before proceeding to apply Circular Hough Transform. The piecewise linear structure of vasculature is defined as a structure formed with multiple such continuous linear shapes having a minimal length $L$ and a maximal width $\mathrm{W}$ with condition $\mathrm{W}<<\mathrm{L}$. These linear shapes are formed using pixels set which includes pixels having almost constant gray level value. this value is lower than non-vessel pixels gray level values in their region and rotating linear structuring element $\mathrm{B}$ is used to detect a linear shape by calculation of statistical variance of pixels gray-level values. B having 1 as width and L1 $>>W$ as length, mathematically this is expressed as

$$
I_{a v}(i, j)=I_{B r}{ }^{M A X}(i . j)
$$

Where, $r=\operatorname{argk}\left(\max \left\{I_{B k}{ }^{V A R}(i . j), k=1, \ldots \ldots, 12\right\}\right)$
$\mathrm{Br}$ is structuring element $\mathrm{B}$ at rotation $\mathrm{r}$ determined using (7). Structuring element having 12 rotations, each $15^{0}$ apart were used. The structuring element length $\mathrm{L}_{1}$ should be selected such a way that it will cross vessels to remove them by applying (6).

\section{E. Determining OD Boundary Candidate}

An Optic Disc boundary differentiates the OD from its background. OD boundary is determined by an abrupt change in values of gray levels. Gray level values representing OD are higher as compared to gray level values representing its surrounding. Therefore, for detection of OD boundary, we can simply measure the gradient magnitude of gray level variations within tiny image portion. Initially, a pixel values unrepresentative of environment are eliminated by applying mean filter. Then a gradient magnitude image (hereafter $\mathrm{I}_{\mathrm{GM}}$ ) is obtained using the Prewitt edge detector. This operator convolves two $3 \times 3$ kernels for approximation of derivative for vertical and horizontal changes in order to estimate image edge and orientation. Finally, values of partial derivative for each pixel are taken in order to obtain the gradient magnitude image. Thus, image $\mathrm{I}_{\mathrm{GM}}$ contains lots of details along the edges, especially, at location and gray level intensity changes with blood vessels erased, OD boundary edges are the most significant edges within gradient image. Thus, image $\mathrm{I}_{\mathrm{GM}}$ undergoes thresholding to obtain binary mask of candidates for OD boundary.

Gray level image threshold is automatically decided by the Otsu method of thresholding by assuming its two sets composition namely the foreground and the background. Further, between-class variance is maximized in order to establish the method optimum threshold Totsu. Threshold is used to obtain a OD boundary candidates first binary mask. it is given by a simple linearization equation

$$
\begin{aligned}
I_{B}(i, j)=\{0 & \text { if } I_{G M}(i, j)<T_{\text {OTSU }} \\
\{1 & \text { if } I_{G M}(i, j)>T_{\text {OTSU }}
\end{aligned}
$$

The noise in image 4 is removed by morphological operations and binary mask is obtained.

$$
I_{B M}(i, j)=\left(I_{B}\right) C^{M I N}(i, j)
$$

Where, $\mathrm{C}$ is circular structuring element with diameter of pixels.

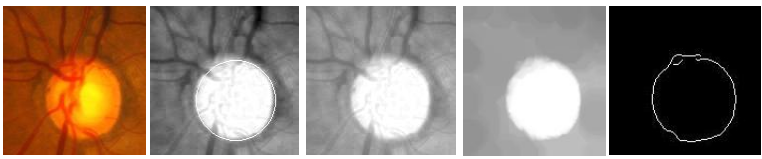

Fig. 4. Illustration of approximation OD boundary

\section{$F$. Final OD Boundary Segmentation}

Circular Hough Transform is used to obtain circular shapes present in the image $\mathrm{I}_{\mathrm{BM}}$ It is written as follows

$$
(P C, r)=\operatorname{CHT}\left(I_{B M}, r_{\text {min }}, r_{\text {max }}\right)
$$

Where, $\mathrm{Pc}=(\mathrm{ic}, \mathrm{jc})$ and $\mathrm{r}$ represents the co-ordinates of center and circular shape radius respectively. These shapes have highest punctuation in the CHT respectively. $r$ is 
limited to the range between $r_{\min }$ and $r_{\max }$ values which are $10 \%$ and $20 \%$ of image divided by two.

\section{Testing and Results}

We tested these methods on the publicly available MESSIDOR data base and NIOP data base that is obtained from Local hospital, National Institute of Ophthalmology, Pune. The algorithm has been applied on 60 images from NIOP database and 100 images from MESSIDOR data base. The evaluation is made against the gold standard of the OD rim that was manually delimited by experts.

Segmentation plays very important role to improve accuracy of final OD boundary detection algorithm. With adaptive histogram equalization we get better segmentation results as compared to other methods and ultimately it gives better OD boundary detection results.

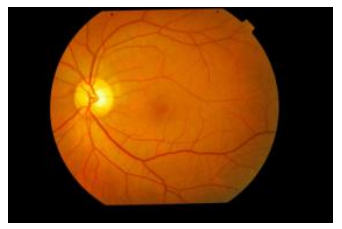

(a)

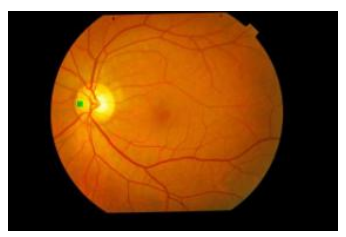

(c)

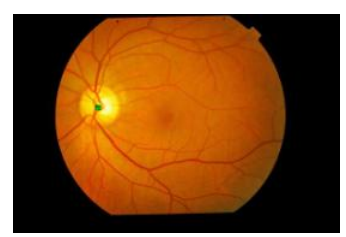

(e)

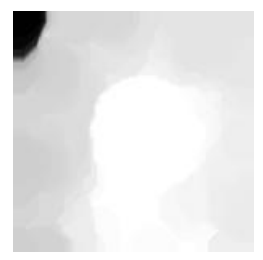

(g)

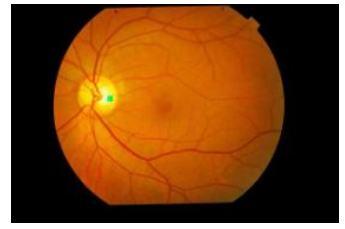

(b)

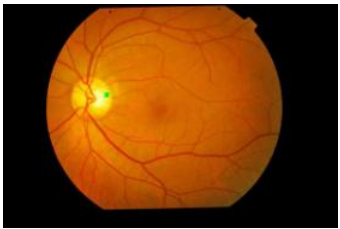

(d)

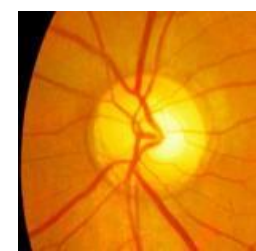

(f)

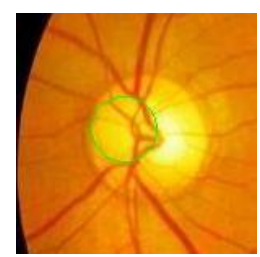

(h)
Fig. 5. Results of the proposed work - (a)Eye Image (b)OD pixel located with maximum difference method (c)OD pixel located with maximum variance method (d)OD pixel located with Low Pass Filter method (e)OD pixel located with Voting algorithm (f) Sub image extracted from retinal image containing OD image

(g)Image with blood vessels erased (h)OD boundary segmentation using Circular Hough Transform.
Proposed works Performance was determined by comparison of OD boundaries in "gold standard" with approximated OD boundaries obtained from proposed algorithm. Overlapping degree between hand-labeled OD region and one segmented by Circular Hough algorithm is more than $90 \%$ of the database images

The results of final OD boundary detection algorithm are effectively improved after preprocessing. These results are illustrated in Fig. 6. In this Figure, (b) shows the OD boundary without preprocessing and using voting type algorithm whereas (c),(d) and (e) represents OD boundary for three processed images respectively. OD Boundary approximation in image $6(\mathrm{~d})$ is very poor as extracted image does not contain entire OD. Fig. 6(d) refers to image enhanced using Histogram Equalization technique suitable to enhance global contrast of image. The adaptive histogram equalization improves upon local contrast of the image and hence indicates significant improvement in the final OD boundary detection that is evident from Fig. 6(e).

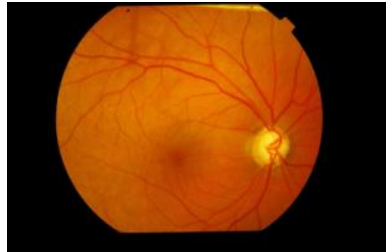

(a)

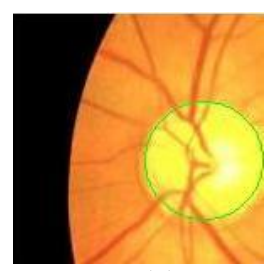

(c)

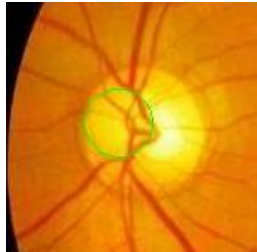

(b)

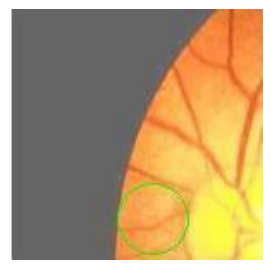

(d)

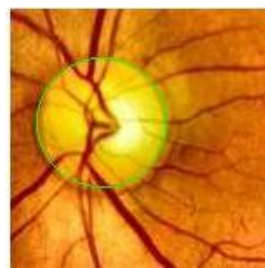

(e)

Fig. 6. OD boundary comparison (a)ODP selection without preprocessing (b)OD Boundary without preprocessing (c)OD boundary in enhanced image (intensity thresholding) (d)OD boundary in enhanced image (histogram equalization) (e)OD boundary in enhanced image (adaptive Histogram eq.).

\section{Conclusion}

Proposed Contrast enhancement method using intensity thresholding technique performs better with maximum variance and Low pass filter methods. Histogram equalization performs better with low pass filter method 
only. Adaptive histogram equalization performs better with all three methods of pixel location and ultimately with voting type algorithm. Adaptive histogram enhance sections of the image, this helps to improve local contrast and effectively improves optic disc boundary segmentation results which in turn improves accuracy of overall method of optic disc detection.

Experimental results on MESSIDOR and NIOP data base proved that the contrast enhancement techniques employed in this research work gives better performance with low pass filter method. It is proposed that complexity in the algorithm can be reduced by using only low pass filter method for OD pixel location in combination with these contrast enhancement techniques with improvement in the accuracy of the final result.

This work is further extended through $\mathrm{C}$ implementation to optimize its mathematical computations and processing time.

We would like to thank Dr. Kelkar and Dr. Bendale of National Institute of Ophthalmology, Pune for sharing with us retinal digital fundus images with their expert guidance.

\section{Reference}

[1] A.Singalavanja,J.Supoavej,P.Bamroongsuk,C.Sinthana yethin,S.Phoojaruenchamachai,and V.Kongbunkiat,'Feasibility study on computer-aided screening for diabetic retinopathy," Jpn. J.Opthalmol.,vol.50.,pp. 361-366,2006.

[2] Arturo Aquino, Manuel Emilio Gegúndez-Arias, Diego Marín" Detecting the Optic Disc Boundary in Digital Fundus Images Using Morphological, Edge Detection, and Feature Extraction Techniques" IEEE Transactions on medical imaging, vol. 29, no.11, November 2010

[3] Shijian Lu," Accurate and Efficient Optic Disc Detection and Segmentation by a Circular Transformation" IEEE Transactions on medical imaging, vol. 30, no.12, November 2011

[4] T.Ashok Kumar, S.Priya, M.G.Mini" Optic Disc Localization in Ocular Fundus Images", International Conference on VLSI, Communication \& Instrumentation (ICVCI) 2011 Proceedings published by International Journal of Computer Applications ${ }^{\circledR}$ (IJCA)

[5] C.Sinthanayothin,"Image analysis for diabetic retinopathy diagnosis,"Ph.D. dissertation, Univ. London, London, U.K., 1999.

[6] A.Hoover and M.Goldbaum,'Locating the optic nerve in a retinal image using fuzzy convergence of the blood vessels,"IEEE Trans.Med.imag.,vol.22,no.8,pp951-958,Aug.2003

[7] A.Hoover and M.Goldbaum,"Fuzzy convergence,"in Proc. IEEE Comput. Soc. Conf. Comput. Vis. Pattern Recognit.,Santa Barbara, CA,1998,PP.716-721

[8] M.Foracchia, E.Grisan , and A.Ruggeri,'Detection of optic disc in retinal images by means of geometrical model of vessel structure,"IEEE Trans.Med.imag.,vol.23,no.10,pp.1189-1195, Oct.2004

[9] A.A.H.A.R.Youssif, A.Z.Ghalwash and A.R.Ghoneim,'Optic disc detection from normalized digital fundus images by means of a vessels' direction matched filter,'IEEE trans. Med. Imag.,vol.27. pp1118,2008 .

[10] A.Osareh,M.Mirmehdi,B.Thomas, and R.Ryder, E.Markham,"Comparison of colour spaces for optic disc localization in retinal images,"Proc. $16^{\text {th }}$ Int. Conf.Pattern Recognit.,2002.pp. 743-746. 